\title{
RIGOROUS DERIVATION OF A NONLINEAR DIFFUSION EQUATION AS FAST-REACTION LIMIT OF A CONTINUOUS COAGULATION-FRAGMENTATION MODEL WITH DIFFUSION
}

\author{
J. A. CARRILLO, L. DESVILLETTES, AND K. FELLNER
}

\begin{abstract}
Weak solutions of the spatially inhomogeneous (diffusive) Aizenmann-Bak model of coagulation-breakup within a bounded domain with homogeneous Neumann boundary conditions are shown to converge, in the fast reaction limit, towards local equilibria determined by their mass. Moreover, this mass is the solution of a nonlinear diffusion equation whose nonlinearity depends on the (size-dependent) diffusion coefficient. Initial data are assumed to have integrable zero order moment and square integrable first order moment in size, and finite entropy. In contrast to our previous result [CDF2], we are able to show the convergence without assuming uniform bounds from above and below on the number density of clusters.
\end{abstract}

\section{INTRODUCTION}

In this work, we shall analyze the fast reaction asymptotics of the spatially inhomogeneous Aizenman-Bak model of coagulation-breakup with spatial diffusion given by

$$
\partial_{t} f-a(y) \triangle_{x} f=Q(f, f) .
$$

Here, $f=f(t, x, y)$ is the concentration of clusters with size $y \geq 0$ at time $t \geq 0$ which are spatially diffusing in $x \in \Omega \subset \mathbb{R}^{d}, d \geq 1$ with normalized volume, i.e., $|\Omega|=1$. Homogeneous Neumann boundary condition:

$$
\nabla_{x} f(t, x, y) \cdot \nu(x)=0 \quad \text { on } \partial \Omega
$$

where $\nu$ denotes the outward unit normal to $\Omega$, are imposed in order to preserve the total number of aggregates. As in [CDF, CDF2], we assume that the diffusion coefficient $a(y)$ is non-degenerate (more precisely, bounded from below and above by strictly positive constants)

$$
0<a_{*} \leq a(y) \leq a^{*}
$$

2000 Mathematics Subject Classification. 35B40, 35Q72, 82D60.

Key words and phrases. fast-reaction limit, coagulation-breakup equation, nonlinear diffusion equations, entropy-based estimates, duality arguments. 
with $a_{*}, a^{*} \in \mathbb{R}^{+}$. The collision operator $Q(f, f)$ takes into account cluster coagulation and fragmentation/breakup, and it reads as

$$
Q(f, f)=Q_{c}(f, f)+Q_{b}(f, f)
$$

with

$$
Q_{c}(f, f):=\int_{0}^{y} f\left(t, x, y-y^{\prime}\right) f\left(t, x, y^{\prime}\right) d y^{\prime}-2 f(t, x, y) \int_{0}^{\infty} f\left(t, x, y^{\prime}\right) d y^{\prime},
$$

and

$$
Q_{b}(f, f):=Q_{b}^{+}(f, f)-Q_{b}^{-}(f, f):=2 \int_{y}^{\infty} f\left(t, x, y^{\prime}\right) d y^{\prime}-y f(t, x, y) .
$$

We refer to [LM, LM2, CDF, CDF2] for a complete set of references and applications in which these models appear.

By noticing that since the reaction term (4) satisfies $\int_{0}^{\infty} y Q(f, f) d y=0$, we have (formally) for all $t \geq 0$ the conservation of total mass,

(5) $\quad N_{\infty}:=\int_{\Omega} N(t, x) d x=\int_{\Omega} N_{i n}(x) d x, \quad$ with $N(t, x):=\int_{0}^{\infty} y f(t, x, y) d y$,

where $\int_{\Omega} N_{\text {in }}(x) d x$ denotes the total inital mass. The corresponding local conservation law writes

$$
\partial_{t} N-\triangle_{x}\left(\int_{0}^{\infty} y a(y) f(t, x, y) d y\right)=0 .
$$

The other important property for eq. (1) is the dissipation of the entropy functional. In order to present it, we first write down the weak form of the operator (4) (for a given test function $\varphi$, and at the formal level):

$$
\begin{aligned}
\int_{0}^{\infty} Q(f, f) \varphi d y= & \int_{0}^{\infty} \int_{0}^{\infty}\left[\varphi\left(y^{\prime}+y\right)-\varphi(y)-\varphi\left(y^{\prime}\right)\right] f(y) f\left(y^{\prime}\right) d y d y^{\prime} \\
& +2 \int_{0}^{\infty} \Phi(y) f(y) d y-\int_{0}^{\infty} y \varphi(y) f(y) d y
\end{aligned}
$$

where the function $\Phi$ is the primitive of $\varphi$ (i.e. $\partial_{y} \Phi=\varphi$ ) such that $\Phi(0)=0$.

Let us consider the entropy functional associated to any positive density $f$ as

$$
H(f)(t, x)=\int_{0}^{\infty}(f \ln f-f) d y
$$

Then, the entropy formally dissipates as

$$
\begin{aligned}
\frac{d}{d t} \int_{\Omega} H(f) d x= & -\int_{\Omega} \int_{0}^{\infty} a(y) \frac{\left|\nabla_{x} f\right|^{2}}{f} d y d x \\
& -\int_{\Omega} \int_{0}^{\infty} \int_{0}^{\infty}\left(f^{\prime \prime}-f f^{\prime}\right) \ln \left(\frac{f^{\prime \prime}}{f f^{\prime}}\right) d y d y^{\prime} d x:=-D_{H}(f),
\end{aligned}
$$

where $f^{\prime \prime}=f\left(t, x, y+y^{\prime}\right), f^{\prime}=f\left(t, x, y^{\prime}\right)$ and $f=f(t, x, y)$. 
Our present aim is to study the fast-reaction asymptotics, i.e. the limit $\varepsilon \rightarrow 0$ of the rescaled problem:

$$
\begin{cases}\partial_{t} f^{\varepsilon}-a(y) \triangle_{x} f^{\varepsilon}=\frac{1}{\varepsilon} Q\left(f^{\varepsilon}, f^{\varepsilon}\right), & \text { for } x \in \Omega, y \in(0, \infty), t>0 \\ \nabla_{x} f^{\varepsilon}(t, x, y) \cdot \nu(x)=0, & \text { for } x \in \partial \Omega, y \in(0, \infty), t>0 \\ f^{\varepsilon}(t=0, x, y)=f_{i n}(x, y) \geq 0, & \text { for } x \in \Omega, y \in(0, \infty)\end{cases}
$$

where we shall assume that the zero- and first-order initial moments as well as the initial entropy are finite, i.e. $\left(1+y+\ln f_{\text {in }}\right) f_{\text {in }} \in L^{1}(\Omega \times(0, \infty))$.

This asymptotic regime is called the fast reaction limit since the reaction term is dominant as $\varepsilon$ gets smaller. In fact, letting formally $\varepsilon \rightarrow 0$, we expect that $f^{\varepsilon} \rightarrow f$, where $Q(f, f)=0$, i.e.

$$
f^{\varepsilon}(t, x, y) \rightarrow e^{-\frac{y}{\sqrt{N(t, x)}}}
$$

where $N(t, x)$ diffuses according to the limit of the moment equation (6):

$$
\partial_{t} N-\triangle_{x} n(N)=0
$$

Here $n(N)$ denotes the function

$$
n(N):=\int_{0}^{\infty} a(y) y e^{-\frac{y}{\sqrt{N}}} d y .
$$

We say that $f^{\varepsilon}$ is a weak solution to eq. (1), see [LM], if $f^{\varepsilon} \in C([0, T], w-$ $\left.L^{1}(\Omega \times(0, \infty))\right)$ such that $y f^{\varepsilon} \in L^{\infty}\left(0, T ; L^{1}(\Omega \times(0, \infty))\right)$ satisfying (1) in the distributional sense with test functions compactly supported in time and in size $y$, and verifying the conservation of mass. Let us remark that global weak solutions to this problem have been constructed in $[\mathrm{LM}]$ satisfying an entropy inequality with a weaker version of the Fisher information as calculated formally in (8):

$$
\begin{aligned}
\int_{\Omega} H\left(f^{\varepsilon}(t)\right) d x & +\left(\int_{0}^{t} \int_{\Omega} \int_{0}^{\infty} a(y)^{1 / 2}\left|\nabla_{x} f^{\varepsilon}\right| d x d y d t\right)^{2}\left(\int_{0}^{t} \int_{\Omega} M^{\varepsilon}(t, x) d x d t\right)^{-1} \\
& +\frac{1}{\varepsilon} \int_{0}^{t} \tilde{D}_{H}\left(f^{\varepsilon}(s)\right) d s \leq \int_{\Omega} H\left(f_{\text {in }}\right) d x
\end{aligned}
$$

for all $t \geq 0$ with

$$
\tilde{D}_{H}(f):=\int_{\Omega} \int_{0}^{\infty} \int_{0}^{\infty}\left(f^{\prime \prime}-f f^{\prime}\right) \ln \left(\frac{f^{\prime \prime}}{f f^{\prime}}\right) d y d y^{\prime} d x
$$


Our main goal is a complete rigorous justification of this formal limit under the following assumptions on the non trivial initial data $f_{\text {in }} \neq 0$ :

$$
\begin{aligned}
& \text { Hypothesis (NL2), } \quad N_{i n}=\int_{0}^{\infty} y f_{i n}(x, y) d y \in L^{2}(\Omega), \\
& \text { Hypothesis (ML1) } \quad M_{i n}=\int_{0}^{\infty} f_{i n}(x, y) d y \in L^{1}(\Omega), \\
& \text { Hypothesis (Entr), } \quad \int_{0}^{\infty} f_{i n}(x, y)\left|\ln \left(f_{i n}(x, y)\right)\right| d y \in L^{1}(\Omega) .
\end{aligned}
$$

The main result of this work is the following:

Theorem 1.1. Let $\Omega$ be a bounded smooth subset of $\mathbb{R}^{d}$ with normalized volume $|\Omega|=1$ and let the diffusion coefficient $a(y)$ satisfy (3). We consider (for all $\varepsilon>0)$ the nonnegative initial data $f^{\varepsilon}(t=0, x, y)=f_{\text {in }}(x, y) \geq 0$ not identically zero satisfying the hypotheses (NL2), (ML1) and (Entr).

Then, as $\varepsilon \rightarrow 0$, the mass $N^{\varepsilon}:=N^{\varepsilon}(t, x)$ associated to any weak solution $f^{\varepsilon}$ to eq. (1) satisfying $(12)$ converges in $L^{1}((0, T) \times \Omega)$ (strongly, and for all $T>0$ ) to the unique solution $N(t, x)$ of the Neumann problem for the nonlinear diffusion equation

$$
\left\{\begin{array}{l}
\partial_{t} N-\triangle_{x} n(N)=0, \\
\left.\nabla_{x} N \cdot \nu(x)\right|_{\partial \Omega}=0,
\end{array}\right.
$$

with initial datum $N_{\text {in }}(0, x)=\int_{0}^{\infty} y f_{\text {in }}(x, y) d y$, with $n(N)$ given by (13).

This result improves our previously published If-Theorem in [CDF2] since it avoids the assumption on the solution that there exists $0<\mathcal{M}_{*} \leq \mathcal{M}^{*}<\infty$ such that $\mathcal{M}_{*} \leq M^{\varepsilon}(t, x)=\int_{0}^{\infty} f^{\varepsilon}(t, x, y) d y \leq \mathcal{M}^{*}$ for all $t \geq 0, x \in \Omega$ and $\varepsilon>0$. These uniform-in- $\epsilon$ bounds, although quite plausible, are not yet known to hold even close to global equilibrium or by linearization techniques applied to problem (9). In fact, any proof would be immediately faced with the difficulties of size-dependent diffusion including possible variants of Turing-type instabilities and diffusion-induced blow-up as known for systems of reaction-diffusion equations.

The presented result is fully by-passing these technical problems providing the first rigorous proof of the fast-reaction limit of a continuous diffusive coagulationfragmentation model. As a trade-off, with the present method we are not able to obtain an estimate on the speed of convergence with respect to the scaling parameter $\varepsilon$.

Our method of proof includes the following ingredients: the estimate of entropy/entropy dissipation as shown in $[\mathrm{CDF}]$ using an inequality proven by Aizenman and Bak for the spatially homogeneous problem in $[\mathrm{AB}]$, a method of duality for parabolic equations as presented, for instance, by M. Pierre and D. Schmitt 
in [PSch], the classical Cziszar-Kullback inequality (cf. [Czi, Kul]), and estimates for the gain of moments taken from [CDF].

Let us comment on the assumptions of Theorem 1.1. Assuming constant coagulation-fragmentation coefficients as in the Aizenman-Bak model [AB] restricts to a particular, yet an important case amongst coagulation-fragmentation models with general detail-balanced coefficients and, thus, with an entropy functional, see e.g. [LM]. Nevertheless, the homogeneous Aizenman-Bak model represents the only case, where a so-called entropy entropy-dissipation estimate is known, i.e. that the entropy dissipation can be controlled below by the relative entropy with respect to local equilibrium states (see estimate (16) below). For general coagulation-fragmentation models an entropy entropy-dissipation estimate is a widely open problem. Let us remark that entropy entropy-dissipation estimates were established in the cases up to four reversible reaction-diffusion equations [DF06, DF], and in case of the discrete homogeneous Becker Döring model [JN].

Moreover, the lower and upper bound on the diffusion coefficient (3) are required for the duality argument. Although there exists a variant of the duality argument avoiding lower bounds of the diffusion coefficients (see, e.g. [CDFpre] in the context of discrete coagulation-fragmentation models) the resulting a-priori bounds are too weak to prove Theorem 1.1 as below.

Finally, we remark that under assumption (3), equation (13) is a wellposed non-degenerate, nonlinear diffusion equation satisfying $0<a_{*} N \leq n(N) \leq a^{*} N$ and $0<a_{*} \leq n^{\prime}(N) \leq a^{*}$, see [LSU] for the existence theory and [CJG, DGJ] for the uniqueness arguments.

Note that fast reactions asymptotics have been rigorously proven in different contexts, cf. [BH] for reversible chemical equations, and [ELM] for discrete coagulation-breakup models. We also refer to [LM, CDF] and the references therein, for studies on the related problem of large time behavior of continuous models of coagulation-fragmentation-diffusion.

Notation 1. We will use various short-cuts like $L_{x}^{p}=L^{p}(\Omega), L_{y}^{p}=L^{p}((0, \infty))$, and $L_{t}^{2}\left(L_{x, y}^{1}\right)=L^{2}\left((0, \infty), L^{1}(\Omega \times(0, \infty))\right)$. Further we denote

$$
M^{\varepsilon}(t, x):=\int_{0}^{\infty} f^{\varepsilon}(t, x, y) d y, \quad N^{\varepsilon}(t, x):=\int_{0}^{\infty} y f^{\varepsilon}(t, x, y) d y,
$$

where $M^{\varepsilon}$ denotes the number density of polymers. The letter $C$ will denote various constants. Moreover $C_{T}$ will indicate a dependence of the constant on the time interval $[0, T]$.

The rest of the paper is devoted to the proof of theorem 1.1. Section 2 consists in proving the basic (independent of $\varepsilon$ ) a-priori estimates for our problem. Then, strong compactness of $M^{\varepsilon}$ and $N^{\varepsilon}$ is obtained in section 3, and moments (up to order 3) are shown to exist in section 4. Finally, the passage to the limit is performed in section 5 . 


\section{A-Priori Estimates}

In this subsection, we prove $\varepsilon$ independent bounds on $M^{\varepsilon}$ and $N^{\varepsilon}$, which will entail compactness of this sequence as shown in the following subsection. We first observe that the conservation law of mass (6) implies

$$
\int_{\Omega} N^{\varepsilon}(t, x) d x=N_{\infty}
$$

for all $t \geq 0$ with $N_{\infty}$ defined in (5).

Moreover, integrating the evolution equation for the monomer density $M^{\varepsilon}$

$$
\partial_{t} M^{\varepsilon}-\triangle_{x}\left(\int_{0}^{\infty} a(y) f^{\varepsilon}(t, x, y) d y\right)=\frac{1}{\varepsilon}\left(N^{\varepsilon}-\left(M^{\varepsilon}\right)^{2}\right),
$$

yields

$$
\frac{d}{d t} \int_{\Omega} M^{\varepsilon}(\tau, x) d x \leq \frac{1}{\varepsilon}\left(N_{\infty}-\left(\int_{\Omega} M^{\varepsilon}(\tau, x) d x\right)^{2}\right)
$$

by Hölder's inequality and (14). Therefore, for all $\varepsilon>0$, we have

$$
\sup _{t \in[0, \infty)} \int_{\Omega} M^{\varepsilon}(t, x) d x \leq \max \left\{\int_{\Omega} M_{i n}(x) d x, \sqrt{N_{\infty}}\right\} .
$$

Entropy Dissipation. The trend to local equilibrium follows from the dissipation of the entropy, which is better understood by using the inequality proven in [AB, Propositions 4.2 and 4.3]: Let $g:=g(y)$ be a function of $L_{+}^{1}((0, \infty))$ with finite entropy $g \ln g \in L^{1}((0, \infty))$, then

$$
\begin{aligned}
\int_{0}^{\infty} \int_{0}^{\infty} g(y) g\left(y^{\prime}\right) \ln g\left(y+y^{\prime}\right) d y d y^{\prime} \leq & \left(\int_{0}^{\infty} g(y) d y\right)\left(\int_{0}^{\infty} g\left(y^{\prime}\right) \ln g\left(y^{\prime}\right) d y^{\prime}\right) \\
& -\left(\int_{0}^{\infty} g(y) d y\right)^{2} .
\end{aligned}
$$

Applying this inequality as in $[\mathrm{CDF}]$ implies that

$$
\int_{0}^{\infty} \int_{0}^{\infty}\left(f^{\prime \prime}-f f^{\prime}\right) \ln \left(\frac{f^{\prime \prime}}{f f^{\prime}}\right) d y d y^{\prime} \geq M H\left(f \mid e^{-\frac{y}{\sqrt{N}}}\right)+2(M-\sqrt{N})^{2},
$$

with the notation $H(f \mid g):=H(f)-H(g)$.

Thus, from the entropy inequality (12) we obtain the estimate

$$
\begin{gathered}
\int_{\Omega} H\left(f^{\varepsilon}(t)\right) d x+\left(\int_{0}^{t} \int_{\Omega} \int_{0}^{\infty} a(y)^{\frac{1}{2}}\left|\nabla_{x} f^{\varepsilon}\right| d x d y d t\right)^{2}\left(\int_{0}^{t} \int_{\Omega} M^{\varepsilon}(t, x) d x d t\right)^{-1} \\
+\frac{1}{\varepsilon} \int_{\Omega}\left[M^{\varepsilon} H\left(f^{\varepsilon} \mid e^{-\frac{y}{\sqrt{N^{\varepsilon}}}}\right)+2\left(M^{\varepsilon}-\sqrt{N^{\varepsilon}}\right)^{2}\right] d x \leq \int_{\Omega} H\left(f_{\text {in }}\right) d x
\end{gathered}
$$


for all $t \geq 0$. Taking into account the Csiszar-Kullback inequality for any two functions $f \geq 0, g>0$ :

$$
\int_{0}^{\infty}\left(f \ln \frac{f}{g}-f+g\right) d y \geq C\left(\int_{0}^{\infty}(f+g) d y\right)^{-1}\left(\int_{0}^{\infty}|f-g| d y\right)^{2},
$$

the dissipation of the entropy (17), together with (3) and (15) imply the uniform bounds

$$
\begin{array}{r}
\sup _{t \in[0, \infty)} \int_{\Omega} \int_{0}^{\infty}\left|f^{\varepsilon} \ln f^{\varepsilon}-f^{\varepsilon}\right| d y d x \leq C, \\
\int_{0}^{T} \int_{\Omega} \int_{0}^{\infty}\left|\nabla_{x} f^{\varepsilon}\right| d y d x d t \leq C_{T}, \\
\int_{0}^{\infty} \int_{\Omega_{\varepsilon}(t)} \frac{M^{\varepsilon}}{M^{\varepsilon}+\sqrt{N^{\varepsilon}}}\left(\int_{0}^{\infty} \mid f^{\varepsilon}-e^{\left.-\frac{y}{\sqrt{N^{\varepsilon}}} \mid d y\right)^{2} d x d t \leq C \varepsilon,}\right. \\
\int_{0}^{\infty} \int_{\Omega}\left|M^{\varepsilon}-\sqrt{N^{\varepsilon}}\right|^{2} d x d t \leq C \varepsilon,
\end{array}
$$

for all $T>0$ with $\Omega_{\varepsilon}(t)=\operatorname{supp}\left(M^{\varepsilon}(t, \cdot)\right)$. We remark that thanks to the a priori bound (21) and the conservation of mass (5), we also deduce a uniform $L^{2}$-bound on $M^{\varepsilon}$, i.e.

$$
\left\|M^{\varepsilon}(t, x)\right\|_{L^{2}((0, T) \times \Omega)} \leq C_{T} .
$$

Duality Argument. Finally, a duality argument (see e.g. [PSch]) implies that solutions of (6), i.e.

$$
\left\{\begin{array}{lc}
\partial_{t} N^{\varepsilon}-\triangle_{x}\left(A^{\varepsilon} N^{\varepsilon}\right)=0, & 0<a_{*} \leq A^{\varepsilon} \leq a^{*}, \\
\left.\nabla_{x} N^{\varepsilon} \cdot \nu(x)\right|_{\partial \Omega}=0, & N^{\varepsilon}(0, x)=N_{i n}(x) \in L^{2}(\Omega),
\end{array}\right.
$$

with

$$
A^{\varepsilon}:=\left\{\begin{array}{cl}
\frac{1}{N^{\varepsilon}} \int_{0}^{\infty} y a(y) f^{\varepsilon}(y) d y & , \text { whenever } N^{\varepsilon}>0 \\
0 & , \text { otherwise }
\end{array}\right.
$$

satisfy a uniform $L^{2}$-bound in terms of the initial data and any $T>0$

$$
\left\|N^{\varepsilon}(t, x)\right\|_{L^{2}((0, T) \times \Omega)} \leq C_{T} .
$$

To show (22), it is known that solutions of the dual parabolic problem

$$
\left\{\begin{array}{l}
-\partial_{t} w-A^{\varepsilon} \triangle_{x} w=H, \quad 0 \leq H \in C_{0}^{\infty}([0, T] \times \Omega) \\
\left.\nabla_{x} w \cdot \nu(x)\right|_{\partial \Omega}=0
\end{array}\right.
$$


with end data $w(T)=0$ and for all non-negative test functions $H \in C_{0}^{\infty}([0, T] \times \Omega)$, satisfy the following $L^{2}$ estimates

$$
\begin{aligned}
\left\|\triangle_{x} w\right\|_{L^{2}([0, T] \times \Omega)} & \leq C\|H\|_{L^{2}([0, T] \times \Omega)}, \\
\|w(0)\|_{L^{2}(\Omega)} & \leq C\|H\|_{L^{2}([0, T] \times \Omega)} .
\end{aligned}
$$

Let us point out that the choice of $L^{2}$ is optimal, i.e. the lowest admissible $L^{p}$ space to have the above estimates for parabolic equation with discontinuous, but bounded coefficients $A^{\varepsilon}$; see counterexamples in [PSch].

On a formal level these estimates are obtained by multiplying the equation in $w$ by $-\Delta_{x} w$, which gives

$$
\begin{aligned}
-\frac{1}{2} \frac{d}{d t} \int_{\Omega}\left|\nabla_{x} w(t)\right|^{2} d x+\int_{\Omega} A^{\varepsilon}\left(\Delta_{x} w\right)^{2} d x & =-\int_{\Omega} H \Delta_{x} w d x \\
& \leq \int_{\Omega}\left[\frac{a_{*}}{2}\left(\Delta_{x} w(t)\right)^{2}+C\left(a_{*}\right) H^{2}\right] d x,
\end{aligned}
$$

where $0<a_{*} \leq A^{\varepsilon}$. After integration in time, it follows that

$$
\int_{0}^{T} \int_{\Omega}\left(\Delta_{x} w\right)^{2} d x \leq C \int_{0}^{T} \int_{\Omega} H^{2} d x
$$

Going back to the equation in $w$, we deduce a bound for $\partial_{t} w$ in $\left.L^{2}((0, T) \times \Omega)\right)$ and therefore a bound $w(0)$ in $L^{2}(\Omega)$ in terms of the $\left.L^{2}((0, T) \times \Omega)\right)$ norm of $H$. Standard approximations allow to render this formal arguments rigorous (cf. [PSch]). The global bound (22) follows finally by duality after integration by parts

$$
\int_{0}^{T} \int_{\Omega} N^{\varepsilon} H d x d t=\int_{\Omega} N_{i n} w(0) d x \leq C\left\|N_{i n}\right\|_{L^{2}(\Omega)}\|H\|_{L^{2}([0, T] \times \Omega)} .
$$

\section{Compactness of $M^{\varepsilon}$ AND $N^{\varepsilon}$}

In this section, we show that the bounds derived in subsection 2 imply a.e. convergence of $M^{\varepsilon}$ and $N^{\varepsilon}$.

Step 1: Using (19) gives

$$
\int_{0}^{T} \int_{\Omega}\left|\nabla_{x} M^{\varepsilon}\right| d x d t \leq \int_{0}^{T} \int_{\Omega} \int_{0}^{\infty}\left|\nabla_{x} f^{\varepsilon}\right| d x d y d t \leq C_{T}
$$

and thus, for all $h \in \mathbb{R}^{d}$ and any domain $\omega \subset \Omega$, such that $h+\omega \subset \Omega$, we obtain

$$
\int_{0}^{T} \int_{\omega}\left|M^{\varepsilon}(t, x+h)-M^{\varepsilon}(t, x)\right| d x d t \leq C_{T}|h| .
$$

Step 2: Let us fix any $h \in \mathbb{R}^{d}$ and any domain $\omega \subset \Omega$, such that $h+\omega \subset \Omega$. As a consequence of (23) and (21) it follows easily that

$$
\int_{0}^{T} \int_{\omega}\left|\sqrt{N^{\varepsilon}}(t, x+h)-\sqrt{N^{\varepsilon}}(t, x)\right| d x d t \leq C_{T}\left(\varepsilon^{\frac{1}{2}}+|h|\right) .
$$


Given any $R>0$, it is straightforward, using the $L^{2}$-bound (22), to check that

$$
\begin{aligned}
& \int_{0}^{T} \int_{\omega}\left|\sqrt{N^{\varepsilon}}(t, x+h)-\sqrt{N^{\varepsilon}}(t, x)\right|\left|\sqrt{N^{\varepsilon}}(t, x+h)+\sqrt{N^{\varepsilon}}(t, x)\right| d x d t \\
& \quad \leq 2 R C_{T}\left(\varepsilon^{\frac{1}{2}}+|h|\right)+\frac{3}{R^{2}} \int_{0}^{T} \int_{\Omega}\left|N^{\varepsilon}\right|^{2}(x) d x d t \leq C_{T}\left[R\left(\varepsilon^{\frac{1}{2}}+|h|\right)+\frac{1}{R^{2}}\right],
\end{aligned}
$$

by dividing the first integral into four different sets, where $\sqrt{N^{\varepsilon}}(t, x)<R$ and $\sqrt{N^{\varepsilon}}(t, x)>R$ and where $\sqrt{N^{\varepsilon}}(t, x+h)<R$ and $\sqrt{N^{\varepsilon}}(t, x+h)>R$, respectively. Optimizing $R$, i.e. choosing $R=\left(\varepsilon^{\frac{1}{2}}+|h|\right)^{-\frac{1}{3}}$, leads to

$$
\int_{0}^{T} \int_{\omega}\left|N^{\varepsilon}(t, x+h)-N^{\varepsilon}(t, x)\right| d x d t \leq C_{T}\left(\varepsilon^{\frac{1}{3}}+|h|^{\frac{2}{3}}\right) .
$$

Step 3: Let us fix now a smooth mollifier $\phi(x) \in C_{0}^{\infty}\left(\mathbb{R}^{d}\right), \phi \geq 1$ with unit integral and support on the euclidean ball centered at 0 of unit radius and $\phi(x)=$ $\phi(-x)$. Let us define for any $\delta>0$, the sequence $\phi_{\delta}(x)=\delta^{-d} \phi\left(\frac{x}{\delta}\right)$.

We first remark that $N^{\varepsilon}$ are solutions of equation (6) in the following weak sense

$$
\int_{\Omega}\left(N^{\varepsilon}(t+\tau, x)-N^{\varepsilon}(t, x)\right) \varphi(x) d x=\int_{t}^{t+\tau} \int_{\Omega} A^{\varepsilon}(s, x) N^{\varepsilon}(s, x) \triangle_{x} \varphi(x) d x d s,
$$

for all $\varphi \in C_{0}^{\infty}\left(\mathbb{R}^{d}\right)$ smooth with $\left.\nabla_{x} \varphi \cdot \nu(x)\right|_{\partial \Omega}=0$ and all $t \geq 0$ and $\tau>0$.

Given any subdomain $\omega$ such that $\bar{\omega} \subset \Omega$, let us consider the characteristic function of the domain $\omega$ denoted by $\chi_{\omega}$, then the function $\phi_{\delta} *\left(\chi_{\omega} \psi\right)$ with $\psi(t, x) \in L^{\infty}((0, T) \times \omega)$ is a suitable test function above since it is a smooth function with $\overline{\operatorname{supp}_{x}(\psi(t, x))} \subset \Omega$ for $\delta$ small enough and a.e. $t \in(0, T)$. Thus, we deduce that for $t \in[0, T]$

$$
\begin{aligned}
& \left|\int_{0}^{T} \int_{\omega}\left(N^{\varepsilon} * \phi_{\delta}(t+\tau, x)-N^{\varepsilon} * \phi_{\delta}(t, x)\right) \psi(t, x) d x d t\right| \\
& \quad \leq \int_{0}^{T} \int_{\omega} \int_{t}^{t+\tau}\left|\left(\int_{0}^{\infty} y a(y) f^{\varepsilon}(s, x, y) d y\right) *\left(\triangle_{x} \phi_{\delta}\right)\right||\psi(t, x)| d s d x d t \\
& \quad \leq \int_{0}^{T} \int_{t}^{t+\tau} a^{*}\left\|N^{\varepsilon}\right\|_{L_{x}^{1}}\left\|\triangle_{x} \phi_{\delta}\right\|_{L_{x}^{1}} d s d t\|\psi\|_{L_{t, x}^{\infty}} \leq C_{T}|\tau|\left\|\triangle_{x} \phi_{\delta}\right\|_{L_{x}^{1}}\|\psi\|_{L_{t, x}^{\infty}} .
\end{aligned}
$$

Using the duality $L^{1}-L^{\infty}$, we finally deduce

$$
\int_{0}^{T} \int_{\omega}\left|N^{\varepsilon} * \phi_{\delta}(t+\tau, x)-N^{\varepsilon} * \phi_{\delta}(t, x)\right| d x d t \leq C_{T}|\tau|\left\|\triangle_{x} \phi_{\delta}\right\|_{L_{x}^{1}} .
$$


Recalling (24) it follows that

$$
\begin{aligned}
\int_{0}^{T} \int_{\omega}\left|N^{\varepsilon}(t+\tau, x)-N^{\varepsilon}(t, x)\right| d x d t \leq & C_{T}|\tau|\left\|\triangle_{x} \phi_{\delta}\right\|_{L_{x}^{1}} \\
& +C_{T} \int_{\mathbb{R}^{d}}\left(\varepsilon^{\frac{1}{3}}+|x|^{\frac{2}{3}}\right) \phi_{\delta}(x) d x .
\end{aligned}
$$

Taking $\delta=|\tau|^{\frac{3}{8}}$ with $\tau$ small enough, we find

$$
\int_{0}^{T} \int_{\omega}\left|N^{\varepsilon}(t+\tau, x)-N^{\varepsilon}(t, x)\right| d x d t \leq C_{T}\left(\varepsilon^{\frac{1}{3}}+|\tau|^{\frac{1}{4}}\right),
$$

and, finally

$$
\int_{0}^{T} \int_{\omega}\left|N^{\varepsilon}(t+\tau, x+h)-N^{\varepsilon}(t, x)\right| d x d t \leq C_{T}\left(\varepsilon^{\frac{1}{3}}+|\tau|^{\frac{1}{4}}+|h|^{\frac{2}{3}}\right) .
$$

Hence, by the Riesz-Frechet-Kolmogorov compactness theorem, the sequence $N^{\varepsilon}$ is strongly compact in $L^{1}((0, T) \times \Omega)$, and so $\sqrt{N^{\varepsilon}}$ is strongly compact in $L^{2}((0, T) \times \Omega)$, and thus, due to $(21), M^{\varepsilon}$ is strongly compact in $L^{2}((0, T) \times \Omega)$. In particular, as $\varepsilon \rightarrow 0$, for a subsequence that we denote with the same index, there exists a $M \geq 0$ and

$$
M^{\varepsilon} \rightarrow M \text { a.e., } \quad N^{\varepsilon} \rightarrow M^{2} \text { a.e., } \quad \text { as } \varepsilon \rightarrow 0,
$$

by the use of $(21)$.

\section{Moment estimate}

We show that solutions of (9) satisfying the hypotheses (NL2) have moments in $y$ up to order three bounded for all $t>0$.

We start by observing [MW, Appendix A] that for given nontrivial initial data $y f_{\text {in }} \in L_{x, y}^{1}$, there exists a concave function $\Phi(r):[0, \infty) \longrightarrow \mathbb{R}$, depending on $f_{\text {in }}$, smoothly increasing in $r$ from $\Phi(0)=0$ to $\infty$ sublinearly, such that

$$
\int_{\Omega} \int_{0}^{\infty} y \Phi(y) f_{\text {in }}(y) d y d x<\infty .
$$

Moreover, the function $\Phi$ can be constructed to grow at most linearly in $y$ and to satisfy

$$
\Phi(y)-\Phi\left(y^{\prime}\right) \geq C \frac{y-y^{\prime}}{y \ln ^{2}(e+y)}
$$

for $0<y^{\prime}<y$ with a constant $C$ not depending on $f_{i n}$. We refer to [MW, Appendix] for all the details of this construction, which adapt to the present situation without essential difficulties by integrating in the spatial variable in their argument. We consider then in a first step the evolution of the moment

$$
M_{1+\Phi}^{\varepsilon}\left(f^{\varepsilon}\right)(t)=\int_{\Omega} \int_{0}^{\infty} y \Phi(y) f^{\varepsilon}(x, y) d y d x .
$$


For the fragmentation part, we use (27) for $0<y^{\prime}<y$ and estimate

$$
\begin{aligned}
\int_{0}^{\infty} y \Phi(y) Q_{b}\left(f^{\varepsilon}\right) d y & =2 \int_{0}^{\infty} \int_{0}^{y} y^{\prime}\left(\Phi\left(y^{\prime}\right)-\Phi(y)\right) d y^{\prime} f^{\varepsilon}(y) d y \\
& \leq-C \int_{0}^{\infty} \frac{1}{\ln (e+y) y} \int_{0}^{y} y^{\prime}\left(y-y^{\prime}\right) d y^{\prime} f^{\varepsilon}(y) d y \\
& =-C \int_{0}^{\infty} \frac{y^{2}}{\ln ^{-2}(e+y)} f^{\varepsilon}(y) d y \leq-C_{\delta} \int_{0}^{\infty} y^{2-\delta} f^{\varepsilon}(y) d y
\end{aligned}
$$

for all $\delta>0$ and a positive constant $C_{\delta}$, where the $(t, x)$-dependence has been dropped for notational convenience. For the coagulation part, Taylor expansion in the weak formulation (7) shows that for a monotone, at most linearly increasing $\Phi$

$$
\left(y+y^{\prime}\right) \Phi\left(y+y^{\prime}\right)-y \Phi(y)-y^{\prime} \Phi\left(y^{\prime}\right) \leq C y y^{\prime},
$$

for a constant $C$.

Altogether, we estimate the evolution of the moment $M_{1+\Phi}^{\varepsilon}\left(f^{\varepsilon}\right)$ to be

$$
\frac{d}{d t} M_{1+\Phi}^{\varepsilon}\left(f^{\varepsilon}\right) \leq-\frac{C_{\delta}}{\varepsilon} M_{2-\delta}^{\varepsilon}\left(f^{\varepsilon}\right)+\frac{C}{\varepsilon} \int_{\Omega}\left(N^{\varepsilon}\right)^{2} d x
$$

where $M_{2-\delta}^{\varepsilon}\left(f^{\varepsilon}\right)$ denotes the moment $\int_{\Omega} \int_{0}^{\infty} y^{2-\delta} f^{\varepsilon}(y) d y$. Then, integrating in time over the interval $[0, T]$ and using the bounds $(22)$, we see that

$$
\int_{0}^{T} M_{2-\delta}^{\varepsilon}\left(f^{\varepsilon}\right) d t<\infty
$$

thus the moment $M_{2-\delta}^{\varepsilon}\left(f^{\varepsilon}\right)$ is finite for a.e. $t \in(0, T]$.

Next, choosing a time $t_{*}>0$ when $M_{2-\delta}^{\varepsilon}\left(f^{\varepsilon}\right)\left(t_{*}\right)<\infty$, and repeating the above (with $\Phi(y)=y^{1-\delta}$ ) shows that $\int_{t_{*}}^{T} M_{3-\delta}^{\varepsilon}\left(f^{\varepsilon}\right) d t<\infty$. The same argument once more with $\Phi(y)=y$ leads then finally to

$$
\int_{t_{*}}^{T} \int_{\Omega} \int_{0}^{\infty} y^{3} f^{\varepsilon}(y) d y<\infty,
$$

where $t_{*}$ can be chosen arbitrarily small.

\section{PASSing to the Limit}

First, let us fix $T>0$. We observe that due to the estimate (20), up to extraction of a subsequence,

$$
\phi_{\varepsilon}(t, x) \frac{M^{\varepsilon}}{M^{\varepsilon}+\sqrt{N^{\varepsilon}}}\left(\int_{0}^{\infty}\left|f^{\varepsilon}-e^{-\frac{y}{\sqrt{N^{\varepsilon}}}}\right| d y\right)^{2} \rightarrow 0 \quad \text { as } \varepsilon \rightarrow 0 \text {, a.e. }
$$

with $(t, x) \in(0, T) \times \Omega$ and $\phi_{\varepsilon}$ the characteristic function of the $\operatorname{set} \operatorname{supp}\left(M^{\varepsilon}\right)$, and moreover, thanks to the convergence (26)

$$
\int_{0}^{\infty}\left|f^{\varepsilon}-e^{-\frac{y}{\sqrt{N^{\varepsilon}}}}\right| d y \rightarrow 0 \quad \text { as } \varepsilon \rightarrow 0 \quad \text { for a.e. } \quad(t, x) \in B_{M},
$$

where $B_{M}:=\operatorname{supp}(M)$ in $(0, T) \times \Omega$. 
Denoting then $h^{\varepsilon}=f^{\varepsilon}-e^{-\frac{y}{\sqrt{N^{\varepsilon}}}}$ we will show that

$$
\int_{0}^{T} \int_{\Omega} \int_{0}^{\infty} y\left|h^{\varepsilon}\right| d y d x d t \rightarrow 0 \quad \text { as } \varepsilon \rightarrow 0 .
$$

First, the conservation of mass (5) implies that $\int_{0}^{t_{*}} \int_{\Omega} \int_{0}^{\infty} y\left|h^{\varepsilon}\right| d y d x d t=\mathrm{O}\left(t_{*}\right)$ independent of $\varepsilon$ and with $t_{*}>0$ arbitrarily small as in (28).

We then estimate the remaining part of (30) in three steps: First, for $R>2$ thanks to (22) and (28) that

$$
\begin{aligned}
\int_{t_{*}}^{T} \int_{\Omega} \int_{|y| \geq R} y\left|h^{\varepsilon}\right| d y d x d t & \leq \int_{t_{*}}^{T} \int_{\Omega} \int_{|y| \geq R} \frac{y^{3}}{R^{2}}\left[f^{\varepsilon}+e^{-\frac{y}{\sqrt{N^{\varepsilon}}}}\right] d y d x d t \\
& \leq \frac{1}{R^{2}}\left(C+\int_{t_{*}}^{T} \int_{\Omega}\left(N^{\varepsilon}\right)^{2} d x d t\right) \leq \frac{C}{R^{2}} .
\end{aligned}
$$

Secondly, for a constant $Q>1$, we observe that $\left|h^{\varepsilon}\right| \geq Q$ implies (due to $0 \leq$ $e^{-\frac{y}{\sqrt{N^{\varepsilon}}}} \leq 1$ ) that $\left|h^{\varepsilon}\right|=h^{\varepsilon}$ and $f^{\varepsilon} \geq Q$. Thus, using (18)

$$
\begin{aligned}
\int_{t_{*}}^{T} \int_{\Omega} \int_{|y| \leq R} y\left|h^{\varepsilon}\right| \mathbf{1}_{\left\{\left|h^{\varepsilon}\right| \geq Q\right\}} d y d x d t & \leq 2 R \int_{t_{*}}^{T} \int_{\Omega} \int_{|y| \leq R} f^{\varepsilon} \mathbf{1}_{\left\{f^{\varepsilon} \geq Q\right\}} d y d x d t \\
& \leq \frac{2 R}{\ln Q} \int_{t_{*}}^{T} \int_{\Omega} \int_{0}^{\infty} f^{\varepsilon}\left|\ln f^{\varepsilon}\right| d y d x d t \leq \frac{C R}{\ln Q} .
\end{aligned}
$$

Hence, we have

$$
\int_{t_{*}}^{T} \int_{\Omega} \int_{0}^{\infty} y\left|h^{\varepsilon}\right| d y d x d t \leq \frac{C}{R^{2}}+\frac{C R}{\ln Q}+R \int_{t_{*}}^{T} \int_{\Omega} \int_{|y| \leq R}\left|h^{\varepsilon}\right| \mathbf{1}_{\left\{\left|h^{\varepsilon}\right| \leq Q\right\}} d y d x d t .
$$

We need to show that

$$
\int_{t_{*}}^{T} \int_{\Omega} \int_{|y| \leq R}\left|h^{\varepsilon}\right| \mathbf{1}_{\left\{\left|h^{\varepsilon}\right| \leq Q\right\}} d y d x d t \rightarrow 0
$$

as $\varepsilon \rightarrow 0$. We decompose this integral in the $(t, x)$ variables onto $B_{M}$ and its complementary set $B_{M}^{c}=([0, T] \times \Omega) / B_{M}$. In the support of $M(t, x)$, we use (29) to get

$$
\int_{|y| \leq R}\left|h^{\varepsilon}\right| \mathbf{1}_{\left\{\left|h^{\varepsilon}\right| \leq Q\right\}} d y \leq \int_{0}^{\infty}\left|h^{\varepsilon}\right| d y \rightarrow 0 \quad \text { as } \varepsilon \rightarrow 0,
$$

for a.e. $(t, x) \in B_{M}$, and moreover, we have the trivial estimate

$$
\int_{|y| \leq R}\left|h^{\varepsilon}\right| \mathbf{1}_{\left\{\left|h^{\varepsilon}\right| \leq Q\right\}} d y \leq Q R \in L^{1}([0, T] \times \Omega),
$$

and Lebegues' dominated convergence implies that

$$
\int_{B_{M}} \int_{|y| \leq R}\left|h^{\varepsilon}\right| \mathbf{1}_{\left\{\left|h^{\varepsilon}\right| \leq Q\right\}} d y d x d t \rightarrow 0 \quad \text { as } \varepsilon \rightarrow 0 .
$$


In the complementary set, we estimate it as

$$
\int_{B_{M}^{c}} \int_{|y| \leq R}\left|h^{\varepsilon}\right| \mathbf{1}_{\left\{\left|h^{\varepsilon}\right| \leq Q\right\}} d y d x d t \leq \int_{B_{M}^{c}}\left(M^{\varepsilon}(t, x)+\sqrt{N^{\varepsilon}}(t, x)\right) d x d t
$$

for which we know that $M^{\varepsilon}(t, x) \rightarrow M=0$ and $\sqrt{N^{\varepsilon}}(t, x) \rightarrow M=0$ a.e. in $B_{M}^{c}$. Since $M^{\varepsilon}(t, x) \rightarrow M(t, x)$ and $\sqrt{N^{\varepsilon}}(t, x) \rightarrow \sqrt{N}(t, x)=M(t, x)$ in $L^{2}((0, T) \times \Omega)$, then

$$
\int_{B_{M}^{c}}\left(M^{\varepsilon}(t, x)+\sqrt{N^{\varepsilon}}(t, x)\right) d x d t \rightarrow 2 \int_{B_{M}^{c}} M(t, x) d x d t=0
$$

as $\varepsilon \rightarrow 0$. Collecting all previous estimates, we have shown (30).

Finally, we consider a weak formulation of

$$
\left\{\begin{array}{l}
\partial_{t} N^{\varepsilon}-\triangle_{x}\left(\int_{0}^{\infty} y a(y) f^{\varepsilon}(t, x, y) d y\right)=0 \\
\left.\nabla_{x} N^{\varepsilon} \cdot \nu(x)\right|_{\partial \Omega}=0
\end{array}\right.
$$

in the sence that for all $\varphi \in C_{0}^{\infty}\left(\mathbb{R}^{d}\right)$ smooth with $\left.\nabla_{x} \varphi \cdot \nu(x)\right|_{\partial \Omega}=0$ and $T>0$

$$
\int_{\Omega}\left(N^{\varepsilon}(T, x)-N_{i n}^{\varepsilon}(x)\right) \varphi(x) d x=\int_{0}^{T} \int_{\Omega}\left(\int_{0}^{\infty} y a(y) f^{\varepsilon}(t, x, y) d y\right) \triangle_{x} \varphi(x) d x d t,
$$

with initial data satisfying the hypotheses (NL2), (ML1), and (Entr). Then, due to the pointwise convergence (26), we know that $N^{\varepsilon} \rightarrow N=M^{2}$ in $L^{1}((0, T) \times \Omega)$. Further, we consider

$$
\int_{0}^{\infty} y a(y) f^{\varepsilon} d y=\int_{0}^{\infty} y a(y) e^{-\frac{y}{\sqrt{N^{\varepsilon}}}} d y+\int_{0}^{\infty} y a(y) h^{\varepsilon} d y
$$

where with (30)

$$
\int_{0}^{T} \int_{\Omega} \int_{0}^{\infty} y a(y)\left|h^{\varepsilon}\right| d y d x d t \leq a^{*} \int_{0}^{T} \int_{\Omega} \int_{0}^{\infty} y\left|h^{\varepsilon}\right| d y d x d t \rightarrow 0 \quad \text { as } \varepsilon \rightarrow 0 .
$$

Therefore, it follows that

$$
\int_{0}^{\infty} y a(y) f^{\varepsilon} d y \rightarrow n(N),
$$

in $L^{1}((0, T) \times \Omega)$ as $\varepsilon \rightarrow 0$, and moreover $N$ is a weak solution of the problem

$$
\left\{\begin{array}{l}
\partial_{t} N-\triangle_{x} n(N)=0, \\
\left.\nabla_{x} N \cdot \nu(x)\right|_{\partial \Omega}=0
\end{array}\right.
$$

in the following sense:

$$
\int_{\Omega}\left(N(T, x)-N_{i n}(x)\right) \varphi(x) d x=\int_{0}^{T} \int_{\Omega} n(N(t, x)) \triangle_{x} \varphi(x) d x d t,
$$


for all $\varphi \in C_{0}^{\infty}\left(\mathbb{R}^{d}\right)$ smooth with $\left.\nabla_{x} \varphi \cdot \nu(x)\right|_{\partial \Omega}=0$ and $T>0$. Note that $\partial_{t} N^{\varepsilon}$ is bounded in a negative Sobolev space in the $x$ variable allowing to define values of $N$ at a given time $T>0$. This concludes the proof of Theorem 1.1.

\section{ACKnowledgements}

JAC acknowledges the support from DGI-MEC (Spain) project MTM200806349-C03-03. KF has partly been supported by the KAUST Investigator Award 2008 of Peter A. Markowich. The authors acknowledge partial support of the trilateral project Austria-France-Spain (Austria: FR 05/2007 and ES 04/2007, Spain: HU2006-0025 and HF2006-0198, France: Picasso 13702TG and Amadeus 13785 UA). LD and KF thank the CRM for the kind hospitality during the preparation of this work.

\section{REFERENCES}

[AB] M. Aizenman and T. Bak, Convergence to equilibrium in a system of reacting polymers, Comm. Math. Phys., 65 (1979), 203-230.

$[\mathrm{BH}]$ D. Bothe, D. Hilhorst, A reaction-diffusion system with fast reversible reaction, J. Math. Anal. Appl. 286(1)(2003), 125-135.

[CDFpre] J. A. Cañizo, L. Desvillettes and K. Fellner, Regularity and mass conservation for discrete coagulation-fragmentation equations with diffusion, preprint.

[CDF] J. A. Carrillo, L. Desvillettes and K. Fellner, Exponential decay towards equilibrium for the inhomogeneous Aizenman-Bak model, Comm. Math. Phys., 278 (2008), 433-451.

[CDF2] J. A. Carrillo, L. Desvillettes and K. Fellner, Fast-Reaction Limit for the Inhomogeneous Aizenman-Bak Model, Kinetic and Related Models, 1 (2008), 127-137.

[CJG] J. A. Carrillo, A. Jüngel and M. P. Gualdani, Convergence of an entropic semidiscretization for nonlinear Fokker-Planck equations in $\mathbb{R}^{d}$, Publ. Mat., 52 (2008), 413-433.

[Czi] I. Csiszár, Eine informationstheoretische Ungleichung und ihre Anwendung auf den Beweis von Markoffschen Ketten, Magyar Tud. Akad. Mat. Kutató Int. Közl. 8 (1963), 85-108.

[DGJ] J. I. Díaz, G. Galiano, and A. Jüngel, On a quasilinear degenerate system arising in semiconductor theory. Part I: existence and uniqueness of solutions, Nonlin. Anal. RWA, 2 (2001), 305-336.

[DF06] L. Desvillettes, K. Fellner, Entropy methods for Reaction-Diffusion Equations: Slowly Growing A-priori bounds Revista Matemática Iberoamericana 24(2) (2008), 407-431.

[DF] L. Desvillettes, K. Fellner, Entropy Methods for Reaction-Diffusion Equations with Degenerate Diffusion Arising in Reversible Chemistry preprint

[ELM] M. Escobedo, P. Laurençot, S. Mischler, Fast reaction limit of the discrete diffusive coagulation-fragmentation equation, Comm. Partial Differential Equations 28(5-6)(2003), 1113-1133.

[JN] P.E. Jabin, B. Niethammer, On the rate of convergence to equilibrium in the BeckerDöring equations, J. Differential Equations 191 (2003), 518-543.

[Kul] S. Kullback, Information Theory and Statistics, John Wiley, (1959).

[LSU] O. A. Ladyzenskaja, V A. Solonnikov and N. N. Ural'ceva, Linear and Quasilinear Equations of Parabolic Type, AMS, Providence, Rhode Island, (1968). 
[LM] P. Laurençot and S. Mischler, The continuous coagulation-fragmentation equation with diffusion, Arch. Rational Mech. Anal., 162 (2002), 45-99.

[LM2] P. Laurençot and S. Mischler, On coalescence equations and related models, survey in "Modeling and Computational Methods for Kinetic Equations" (eds. P. Degond, L. Pareschi and G. Russo), Model. Simul. Sci. Eng. Technol., Birkhäuser Boston, Boston, MA, (2004), 321-356.

[MW] S. Mischler, B. Wennberg, On the spatially homogeneous Boltzmann equation, Ann. Inst. H. Poincaré Anal. Non Linéaire 16(4) (1999), 467-501.

[PSch] M. Pierre and D. Schmitt, Blowup in reaction-diffusion systems with dissipation of mass., SIAM Rev., 42 (2000), 93-106.

J. A. Carrillo

Icrea (Institució Catalana de Recerca i Estudis Avançats)

and Departament de Matemàtiques

Universitat Autònoma de BARCELONA

E-08193 Bellaterra, Spain

E-mail address: carrillo@mat.uab.es

L. Desvillettes

Cmla, Ens Cachan, Iuf \& Cnrs, Pres UniverSud

61 Av. Du Pdt. Wilson

94235 Cachan Cedex, France

E-mail address: desville@cmla.ens-cachan.fr

K. Fellner

DAmTP, CMs, University OF CAMBridge

WILBERFORCE ROAD

CAmbridge CB3 0WA, United Kingdom

ON LEAVE FROM:

FACULTY OF MATHEMATICS

UNIVERSITY OF VIENNA

NordBergstr. 15, 1090 Wien, AustriA

E-mail address: K.Fellner@damtp.cam.ac.uk

E-mail address: Klemens.Fellner@univie.ac.at 